\title{
Análisis de la efectividad de Lego en la enseñanza de matemáticas en la etapa de Educación Infantil
}


I. Resumen

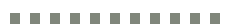

La presente investigación ${ }^{1}$ tiene como principal objetivo analizar la efectividad de Lego para la enseñanza de matemáticas en el alumnado de Educación Infantil. Se ha llevado a cabo una investigación cuasiexperimental con pretest y postest sobre una única muestra, constituida por los 23 alumnos que asisten a una misma aula mixta de Educación Infantil de 3 años. Para comprobar la efectividad, se han realizado tres intervenciones, consistentes cada una de ellas en un test inicial de diagnóstico (pretest), seguido de tres sesiones de juego y aprendizaje con Lego y un test final (postest) para evaluar el cambio. En estas intervenciones, se han trabajado diferentes conceptos matemáticos adecuados a su edad, los cuales todavía no se habían enseñado o, si se habían trabajado, todavía no habían sido adquiridos por las niñas/os. Los conceptos trabajados fueron: a) en la primera intervención, la seriación; b) en la segunda intervención, los números 1,2 y 3 , la grafía del 1 y 2 , y el concepto de cantidad del número 1 y $2 ;$ y c) en la tercera intervención, el concepto de direccionalidad. Los resultados globales obtenidos después de aplicar la prueba de signos (estadística no paramétrica) muestran diferencias estadísticamente significativas que confirman que el alumnado ha aprendido con el uso del Lego.

Palabras claves: Lego Educación, matemáticas, educación infantil, innovación educativa, aprendizaje significativo.

\section{Introducción}

La educación necesita procesos de innovación para transformar los métodos tradicionales que se han quedado anticuados e ir adaptándose a la actualidad. Según Carbonell $(2006,14)$ : «la nueva ciudadanía que hay que formar exige desde los primeros años de la escolarización otro tipo de conocimiento y una participación más activa del alumnado en el proceso de enseñanza». Ya no solo se da valor a los conocimientos básicos como son, por ejemplo, saber escribir y conocer los números o los colores; ahora la nueva ciudadanía necesita aprender a conocer, aprender a hacer, aprender a ser y aprender a convivir. Estos son los cuatro pilares básicos que la UNESCO (1996) ha definido en su informe Delors.

Las personas más indicadas para señalar problemas y hacer posible una mejora son los docentes. Por eso, las innovaciones que parten desde abajo, desde este colectivo, tienen más posibilidades de éxito y continuidad que las que emanan desde arriba, según

\footnotetext{
${ }^{1}$ Quiero expresar mi agradecimiento a Miguel Ángel Fortea Bagán, mi tutor del Trabajo de Final de Grado, por su ayuda y sus aportaciones en esta investigación.
} 
Carbonell (2006). Es muy importante, para mejorar, que los docentes tengan ganas y dedicación.

Donde el alumnado pasa casi todo el tiempo que invierte en la escuela es en el aula. Así, el docente es el principal encargado de la educación de las niñas/os y quien debe tener y buscar las mejores herramientas para su educación, tanto académica como personal. Sobre todo en Infantil, el aprendizaje mediante el juego se hace fundamental, ya que la mayoría del tiempo lo pasan jugando. El juego no consiste únicamente en pasárselo bien y divertirse, sino que, a través de él, se transmiten valores y normas de conducta, se resuelven conflictos, se educa a sus miembros jóvenes y se desarrollan muchas facetas de su personalidad (López 2010).

Carbonell (2006) apunta que algunos pensadores como Platón y Aristóteles ya daban importancia al hecho de aprender mediante el juego y, por otra parte, psicólogos de gran prestigio como Piaget y Vygotsky también hacían especial mención al juego como una actividad fundamental para la niña/o. Piaget destacaba tanto en sus escritos teóricos como en sus observaciones clínicas la importancia del juego como proceso de desarrollo, mientras que Vygotsky caracterizaba el juego como el inicio del comportamiento conceptual o guiado por las ideas. Son muchos los autores que están a favor del juego como actividad principal y fundamental en el desarrollo total de las niñas/os, especialmente en infantil.

Para que se produzca un aprendizaje, la niña/o debe ser partícipe y protagonista en su totalidad. Es así cuando asimila el aprendizaje y lo interioriza. "Manipulando los materiales, los resortes de los juguetes o la ficción de los juegos simbólicos, la niña/o se siente autor, capaz de modificar el curso de los acontecimientos» (López 2010).

Según el sistema de juguetes ESAR, desarrollado por Denise Garon, Rolande Filion y Manon Doucet (1996), se encuentran, dentro de la clasificación de los juguetes, los juguetes de armar, ensamblaje o construcción. Nuestra investigación se va a centrar concretamente en el material de Lego. Este material es mundialmente conocido ya que, desde 1958, Ole Kirk dio a conocer por primera vez el famoso bloque de Lego, que ahora todo el mundo conoce. Este material ofrece la posibilidad de crear infinidad de construcciones y poder pasar horas y horas construyendo, pero no solo ofrece diversión, sino que también se trabajan y fomentan diferentes capacidades. Lamoyi (2012), en su artículo "La robótica Lego Mindstorms ${ }^{\circledR}$ : un recurso didáctico para fortalecer el pensamiento lógico matemático», muestra los diferentes beneficios, tanto inmediatos como a largo plazo, en los alumnos que utilizan Lego basándose en investigaciones anteriores realizadas por Lego Mindstorms ${ }^{\circledR}$ Education. Los beneficios son los siguientes: se involucran activamente en su propio proceso de aprendizaje; desarrollan sus intereses en matemáticas; 
potencian sus habilidades de investigación y resolución de problemas, así como lectura, escritura, habilidades de presentación y creatividad; se forman pensadoras autónomas que, además, son capaces de apreciar el valor de la automotivación y de sentirse con recursos; se convierten en autodidactas activas, y; se fomenta la habilidad para resolver los problemas mediante estrategias centradas en el razonamiento lógico y analítico, así como en el pensamiento crítico.

El presente trabajo hace hincapié en el beneficio del conocimiento matemático. En infantil, el material de Lego es muy común en las aulas, pero como material de juego y distracción. Sin embargo, se puede utilizar para el aprendizaje matemático, ya que resulta muy atractivo y motivador para las más pequeñas.

\section{Objetivos}

El objetivo general de esta investigación es el análisis de la efectividad de Lego en la enseñanza de matemáticas durante la etapa de Educación Infantil.

El problema del que parte esta investigación surge a partir de la conclusión, tras realizar las prácticas en el Grado en Educación Infantil, de que, en la mayoría de las aulas de Educación Infantil, se utilizan materiales tradicionales para la enseñanza de las matemáticas. Por este motivo, la elección de la efectividad de Lego en la enseñanza de matemáticas como objeto de investigación se debe a que es un material atractivo para el alumnado e innovador en este campo, pues en la mayoría de las aulas hay una caja de construcciones Lego o similar que se utiliza únicamente para los tiempos de juego.

\section{Material y método}

Esta investigación-acción está dirigida a un grupo seleccionado de forma no aleatoria. Este grupo es un aula mixta de infantil de 3 años en la que hay un total de 23 sujetos, 11 niñas y 12 niños, con un sujeto que presenta necesidades educativas especiales (NEE).

Se ha hecho un diseño cuasi experimental con pretest y postest a una única muestra. Durante la investigación, se ha realizado un total de tres pruebas. Cada una de estas pruebas contaba con un test inicial de diagnóstico (pretest), tres sesiones de intervención con Lego y su respectivo test final (postest). Los conocimientos que se iban a trabajar en las pruebas se eligieron porque todavía no se habían enseñado en el aula o porque, después de trabajarlos varias veces, los alumnos aún no los habían adquirido según la evaluación de la maestra del grupo. Tampoco se debían estar trabajando en el momento de la intervención. 
El primer concepto que se trabajó fue la seriación. Aunque se había tratado en el aula, todavía no se había adquirido por la mayoría del alumnado y en ese momento no se estaba trabajando.

La prueba tuvo una duración de una semana. Al comienzo de la semana se pasó únicamente una prueba inicial o pretest con el fin de saber qué cantidad de alumnado tenía el concepto adquirido. Antes de pasar la prueba, se explicó una sola vez en qué consistía. Los siguientes tres días, los alumnos trabajaron con el material de Lego por mesas y en equipo para hacerlo de forma colectiva con el resto de sus compañeros de mesa.

En la primera sesión de intervención, se repartió una tarjeta por mesa con la seriación rojo-amarillo y los bloques de Lego Duplo exactos para realizar la seriación. Antes de que ellos comenzaran, se les repitió dos veces la seriación para que la interiorizaran. Esta prueba tuvo una duración de tres minutos para que entre ellos pudieran debatir y hacer la seriación. Una vez pasado el tiempo establecido, se observó el resultado final de la seriación y se les retiró el material.

En la segunda sesión de intervención, el procedimiento y la duración de la prueba fue igual que en la primera sesión, excepto por el número de veces que se verbalizó la seriación previamente a la puesta en marcha de la prueba por parte del alumnado; en este caso se repitió una vez.

En la tercera sesión de intervención, se repartió una tarjeta por mesa con la seriación, los bloques exactos rojos y amarillos, y tres más de otro color. Esta vez no se verbalizó la seriación para observar si con los ladrillos que se habían añadido de más seguirían utilizando únicamente los rojos y los amarillos o los introducirían en la seriación. Esta prueba tuvo la misma duración que las anteriores, con el fin de darles tiempo para debatir y hacer la seriación. Una vez transcurrido el tiempo, se observó lo que habían hecho con los ladrillos de más, se examinó el resultado final de la seriación y, finalmente, se retiró el material.

En la prueba final o postest, se les volvió a pasar la misma prueba que al principio y no se verbalizó ningún tipo de recordatorio sobre la seriación.

\section{Prueba 2}

En esta prueba, se trabajó el reconocimiento de los números 1, 2 y 3; las grafías del 1 y el 2 y, por último, el concepto de cantidad de los números 1 y 2 . Estos conceptos ya se habían introducido en el aula, pero el de cantidad todavía no se había adquirido por la mayoría del alumnado y en ese momento no se estaba trabajando. La duración y la organización de la sesión de intervención han sido 
iguales que en la anterior, a fin de que no hubiera variaciones en la metodología.

La prueba inicial de diagnóstico o pretest se realizó por mesas, con seis alumnas/os como máximo, y consistió en lo siguiente:

1. Para evaluar el reconocimiento de los números, se les pidió que señalaran o cogieran los números construidos con bloques de Lego Duplo que habían encima de la mesa.

2. Para la evaluación del concepto de cantidad relacionado con los números, se les evaluó mediante dos actividades: la actividad de la torre o la actividad de la carretera. Para la realización de estas actividades se les explicó una vez en qué consistía cada actividad, ya que no las habían realizado con anterioridad.

3. Para evaluar si sabían plasmar las grafías de los números, se les pidió que escribieran estos dos números que se iban a trabajar en un folio.

En la primera sesión de intervención, a la hora de los juegos de mesa estipulada en el horario de aula, se les ofreció jugar a las actividades que integraron en la prueba inicial de diagnóstico. Reconocían los números constantemente en las demás actividades, así que esta prueba se centró más en el concepto de cantidad mediante al juego de la torre y la carretera. La actividad de la torre consiste en visualizar los números que están en la tarjeta y depositar encima de cada número, en forma de torre, la cantidad que le corresponde a cada uno de ellos mediante bloques de Lego. Por otro lado, la actividad de la carretera es similar a la de la torre, pero en este caso los bloques de Lego deben colocarse al lado del número correspondiente en forma de carretera. Por último, también se trabajó la grafía con las tarjetas de los números. En estas tarjetas, se deben depositar en primer lugar los ladrillos de Lego siguiendo el recorrido del número, y después escribir en el recuadro el número que se está trabajando en dicha tarjeta.

La segunda sesión de intervención se llevó a cabo la misma hora de juego que en la anterior. Se fue llamando a las niñas/os por grupos para seguir trabajando. En un principio, se convocó a las niñas/os que tenían mayor dificultad en esta prueba o que no habían adquirido los conceptos básicos que se iban a trabajar, como es el reconocimiento de los números. En esta segunda sesión, se trabajó con toda la clase y todos realizaron las mismas actividades, pero no todos invirtieron el mismo tiempo en realizarlas. Durante la sesión se utilizó la misma metodología que en la primera sesión, pero en esta se empezó trabajando la grafía de los números, se continuó con el concepto de cantidad y se finalizó con el reconocimiento de los números. 
La tercera sesión de intervención se realizó en la hora del juego libre estipulado en el horario de aula. Se fue llamando por grupos al alumnado para seguir trabajando los conceptos de esta prueba. La metodología que se utilizó fue la misma que los días anteriores, igual que las actividades que se realizaron en esta última sesión.

La prueba final o postest se realizó de forma individual y aislada del grupo para no contaminar el resultado por la influencia del resto de la muestra. En esta prueba se realizaron las mismas actividades que a lo largo de la intervención, pero no se ofreció ayuda ni se verbalizó ninguna indicación.

\section{Prueba 3}

En la última prueba, se trabajó el concepto de la direccionalidad (delante, detrás, a un lado). Este concepto todavía no se había trabajado en el aula. Tanto la duración como la metodología de esta prueba son iguales que en las dos anteriores.

Al comienzo de la semana, se realizó la prueba inicial de diagnóstico para evaluar el conocimiento que tenían sobre el concepto de direccionalidad (delante, detrás y a un lado). La prueba consistió en colocar, según las indicaciones que se iban dando, un muñeco delante o detrás o a un lado, teniendo como referencia un muro de Lego.

En la primera sesión de intervención, se construyó un muro pequeño con los ladrillos del set de ladrillos Lego Soft en una zona espaciosa. Este muro se utilizó como referencia en la actividad para trabajar la direccionalidad. Para esta sesión, se llamó al alumnado según el grupo de mesa que tienen establecido en el aula. Los grupos de mesas están compuestos por un total de cinco o seis alumnos. Se les explicaron los diferentes conceptos comparándolos con su cuerpo: la parte de delante del muro, que estaba señalizada mediante un dibujo, se comparó con la parte frontal del cuerpo que es la cara y la barriga; la parte de detrás del muro se comparó con la parte trasera del cuerpo que es la espalda, y el concepto a un lado del muro se comparó con las extremidades superiores del cuerpo, los brazos. Una vez finalizada la explicación, los alumnos fueron saliendo uno a uno para poder realizar la actividad. La actividad de esta prueba consistió en que el alumnado, por medio de la experimentación con su propio cuerpo, se fuera colocando según las indicaciones que se dándole daban, teniendo como referencia el muro construido con Lego. En esta sesión se ofrecieron ayudas mediante gestos para que pudieran experimentar y aprender los conceptos sobre la direccionalidad. Durante la actividad se fueron diciendo las tres posiciones que se iban a trabajar, sin repetirlas.

La segunda sesión de intervención se realizó en la misma zona que la sesión anterior. En ella, construyó un muro más grande que el primero. Se fue llamando por mesas al alumnado para que fueran 
realizando la actividad. En primer lugar se repasaron los conceptos

que se iban a trabajar $y$, posteriormente, de forma individual, el alumnado fue haciendo la actividad teniendo en cuenta las indicaciones que se habían dado sobre su posición con respecto al muro. Durante la actividad se fueron verbalizando en orden y repetidamente las diferentes directrices.

La tercera sesión de intervención se realizó en otra zona diferente del aula para cambiar la zona de referencia que tenían de las anteriores sesiones. En esta ocasión se construyó una torre alta orientada de manera diferente a las demás para evitar que se acostumbrasen a una misma posición y construcción. Las indicaciones que se les dieron tenían un orden diferente al de la sesión anterior y se repitieron diversas indicaciones para asegurar el aprendizaje del concepto, no la memorización.

Por último, para la evaluación de la prueba, se construyó un puente y se ubicó en la misma zona que la prueba inicial de diagnóstico, pero con una orientación distinta. Se fue llamando uno por uno a los alumnos para llevar a cabo una evaluación individualizada, sin influencia de los demás. Durante la prueba verbalizaron las diferentes posiciones, como anteriormente, en diferente orden y sin repeticiones.

\section{Resultados}

La muestra de esta investigación estaba compuesta de un total de 23 sujetos, pero, a causa de las ausencias en alguna de las pruebas, se ha reducido a 19 sujetos.

Todas las pruebas han sido calificadas de la misma manera: los sujetos que completaron correctamente la prueba recibieron una calificación de 1 , mientras que cuando no la completaron o lo hicieron de forma incorrecta, la calificación fue un 0 .

Para verificar la significación de las pruebas se ha utilizado la prueba de McNemar, la cual sirve para ver las diferencias en una muestra de pequeño tamaño que requiere de estadística no paramétrica.

\section{Prueba 1}

Como se muestra en la tabla 1 , en la prueba inicial de diagnóstico, 13 sujetos completaron bien la prueba y 6 que lo hicieron incorrectamente. Por otro lado, en la prueba final, 14 completaron correctamente la prueba y 5 de forma incorrecta. También podemos observar que 2 sujetos no han aprendido nada, ya que no superaron la prueba inicial ni la final; por otra parte, 3 sujetos han empeorado, pues completaron correctamente la primera prueba, pero no la segunda. 
Aplicando la prueba de McNemar, se deduce que no ha habido una mejora significativa, ya que la probabilidad de dos colas es superior a 0,001, lo cual no supone una diferencia estadísticamente significativa.

Tabla 1. Resultados prueba 1

\begin{tabular}{|c|c|c|c|c|c|}
\hline \multirow{2}{*}{ Alumnado } & \multicolumn{2}{|c|}{ Prueba 1 } & Alumno 10 & $\mathbf{0}$ & $\mathbf{1}$ \\
\cline { 2 - 6 } & P. I. & P. F. & Alumno 11 & 0 & 1 \\
\hline Alumno 1 & 1 & 1 & Alumno 12 & 0 & 1 \\
\hline Alumno 2 & 0 & 0 & Alumno 13 & 0 & 0 \\
\hline Alumno 3 & 1 & 1 & Alumno 14 & 0 & 1 \\
\hline Alumno 4 & 1 & 1 & Alumno 15 & 1 & 1 \\
\hline Alumno 5 & 1 & 1 & Alumno 16 & 1 & 1 \\
\hline Alumno 6 & 1 & 1 & Alumno 17 & 1 & 1 \\
\hline Alumno 7 & 1 & 0 & Alumno 18 & 1 & 1 \\
\hline Alumno 8 & 1 & 0 & Alumno 19 & 1 & 1 \\
\hline Alumno 9 & 1 & 0 & Total & 13 & 14 \\
\hline
\end{tabular}

\section{Prueba 2}

Como se observa en la tabla 2, en la prueba de diagnóstico hay un total de 6 sujetos que la han completado correctamente y 13 de forma incorrecta. En la prueba final, hay 10 sujetos que la han superado y otros 9 que no. En esta prueba se sigue observando que hay 2 sujetos que no completaron satisfactoriamente el pretest y siguieron sin hacerlo al someterlos al postest. En cambio, solo hay un sujeto que ha empeorado.

Aplicando la prueba de McNemar, se extrae que no ha habido una mejora significativa: la probabilidad de dos colas es superior a 0,001 , lo cual no se considera una diferencia estadísticamente significativa.

Tabla 2. Resultados prueba 2

\begin{tabular}{|c|c|c|c|c|c|}
\hline \multirow{2}{*}{ Alumnado } & \multicolumn{2}{|c|}{ Prueba 2 } & Alumno 10 & $\mathbf{1}$ & $\mathbf{0}$ \\
\cline { 2 - 6 } & P. I. & P. F. & Alumno 11 & 0 & 0 \\
\hline Alumno 1 & 1 & 1 & Alumno 12 & 0 & 1 \\
\hline Alumno 2 & 0 & 0 & Alumno 13 & 0 & 0 \\
\hline Alumno 3 & 0 & 0 & Alumno 14 & 0 & 0 \\
\hline Alumno 4 & 0 & 1 & Alumno 15 & 0 & 1 \\
\hline
\end{tabular}




\begin{tabular}{|c|c|c|c|c|c|}
\hline Alumno 5 & 0 & 0 & Alumno 16 & 1 & 1 \\
\hline Alumno 6 & 1 & 1 & Alumno 17 & 0 & 1 \\
\hline Alumno 7 & 1 & 1 & Alumno 18 & 1 & 1 \\
\hline Alumno 8 & 0 & 1 & Alumno 19 & 0 & 0 \\
\hline Alumno 9 & 0 & 0 & $\begin{array}{c}\text { Total } \\
\text { aciertos }\end{array}$ & 6 & 10 \\
\hline
\end{tabular}

\section{Prueba 3}

Se observa en la tabla 3 que solo tres sujetos han completado correctamente la prueba inicial, mientras que 17 no lo han hecho. Por otro lado, 14 sujetos han superado la prueba final, pero 5 no lo han hecho.. En este caso solo hay 2 sujetos que no han aprendido nada después de realizar toda la intervención.

Aplicando la prueba de McNemar, en este caso sí observamos una mejora significativa, ya que la probabilidad de dos colas es 0,0005 . Esta cifra sí constituye una diferencia estadísticamente significativa al $99 \%$.

Tabla 3. Resultados prueba 3

\begin{tabular}{|c|c|c|c|c|c|}
\hline \multirow{2}{*}{ Alumnado } & \multicolumn{2}{|c|}{ Prueba 3 } & Alumno 10 & $\mathbf{1}$ & $\mathbf{1}$ \\
\cline { 2 - 6 } & P. I. & P. F. & Alumno 11 & 0 & 0 \\
\hline Alumno 1 & 0 & 1 & Alumno 12 & 0 & 1 \\
\hline Alumno 2 & 0 & 0 & Alumno 13 & 0 & 0 \\
\hline Alumno 3 & 0 & 1 & Alumno 14 & 0 & 1 \\
\hline Alumno 4 & 0 & 1 & Alumno 15 & 0 & 1 \\
\hline Alumno 5 & 0 & 0 & Alumno 16 & 0 & 1 \\
\hline Alumno 6 & 0 & 1 & Alumno 17 & 1 & 1 \\
\hline Alumno 7 & 0 & 1 & Alumno 18 & 0 & 1 \\
\hline Alumno 8 & 0 & 0 & Alumno 19 & 0 & 1 \\
\hline Alumno 9 & 0 & 1 & Total & 2 & 14 \\
\hline
\end{tabular}

\section{Prueba global}

Por último, se observa en la tabla 4 el total de puntos acumulado de las 3 pruebas. En primer lugar, en el total de puntuación de la prueba inicial de diagnóstico, se obtiene una puntuación de 21 sobre 57. En segundo lugar, en el total de puntuación de la prueba final, obtenemos un resultado de 38 sobre 57. 
Aplicando la prueba de los signos, se extrae un valor t de 4,46 que con 18 grados de libertad da una probabilidad de 2 colas de 0,000 . Esto denota que existen diferencias significativas, de forma que en la prueba final se demuestra que sí han aprendido con el Lego.

Tabla 4. Resultados totales de las pruebas

\begin{tabular}{|c|c|c|c|c|c|}
\hline \multirow{2}{*}{ Alumnado } & \multicolumn{2}{|c|}{ Total } & Alumno 10 & $\mathbf{2}$ & $\mathbf{2}$ \\
\cline { 2 - 6 } & Inicial & Final & Alumno 11 & 0 & 1 \\
\hline Alumno 1 & 2 & 3 & Alumno 12 & 0 & 3 \\
\hline Alumno 2 & 0 & 0 & Alumno 13 & 0 & 0 \\
\hline Alumno 3 & 1 & 2 & Alumno 14 & 0 & 2 \\
\hline Alumno 4 & 1 & 3 & Alumno 15 & 1 & 3 \\
\hline Alumno 5 & 1 & 1 & Alumno 16 & 2 & 3 \\
\hline Alumno 6 & 2 & 3 & Alumno 17 & 2 & 3 \\
\hline Alumno 7 & 2 & 2 & Alumno 18 & 2 & 3 \\
\hline Alumno 8 & 1 & 1 & Alumno 19 & 1 & 2 \\
\hline Alumno 9 & 1 & 1 & Total & 21 & 38 \\
\hline
\end{tabular}

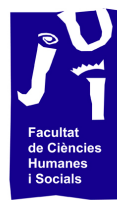

UNIVERSITAT JAUME•I

Prueba de signos

$\begin{array}{ccc}\text { t-value } & \mathrm{df} & 2-\text { tail } \mathrm{Sig} \\ 4,46 & 18 & , 000\end{array}$

\section{Discusiones y conclusiones}

El objetivo principal de esta investigación era analizar la efectividad del material de Lego en la enseñanza de las matemáticas $y$, después de haber observado los resultados, se puede concluir que Lego sí es un material con el que el alumnado ha aprendido y sirve para enseñar conceptos matemáticos en la etapa de Educación Infantil.

En cuanto al tema del aprendizaje del alumnado, se puede advertir en las diferentes tablas de resultados que ha habido resultados distintos en cuanto al aprendizaje a lo largo de las tres pruebas. Uno de los resultados que debemos destacar en cuanto al aprendizaje es que ha habido dos sujetos, más concretamente el alumno 2 y el alumno 13, que durante las tres pruebas no han aprendido nada, han partido sin conocer el concepto y han finalizado igual. Otro resultado que conviene mencionar es que determinados 
sujetos conocían al principio el concepto evaluado, pero terminan la prueba sin saberlo. Este caso se ha producido en cuatro alumnos diferentes, tanto en la prueba 1 como en la prueba 2. Por último, cabe mencionar el resultado en cuanto al aprendizaje de los sujetos que han empezado la prueba sin saber el concepto y la terminan con el conocimiento adquirido. Este resultado es el que ha predominado en la mayoría de las pruebas; por ello, se puede concluir que ha habido una diferencia significativa y un aprendizaje del alumnado. En relación con los resultados en los que no se aprecia aprendizaje o incluso se observa una involución, pueden haber estado influidos por muchos factores externos a las pruebas y se podría justificar esa ausencia de aprendizaje. Entre los factores que han podido influir en estos diferentes resultados, están el interés o la motivación; si los sujetos no estaban motivados o no mostraban interés hacia las pruebas propuestas, era imposible que obtuvieran ese conocimiento.

A continuación, en cuanto a las pruebas que se han utilizado para esta investigación, la prueba 1 se evaluó mediante una ficha en la que aparecían seis piezas de Lego, 3 con la serie rojo-amarillo y 3 blancas para continuarla. De esta prueba hay diversos aspectos que se pueden valorar y mejorar. En primer lugar, debemos mencionar que la ficha, al constar de seis ladrillos en su totalidad, abre la posibilidad de error. Con esto nos referimos a que, al tener tres ladrillos en blanco, los alumnos pueden dudar si tienen que seguir la serie o empezarla. En consecuencia, sería más conveniente poner un total de 5 ladrillos a fin de minimizar esa posibilidad de error. Por otro lado, se observa en los resultados que ha sido la prueba donde menos aprendizaje se ha producido, ya que los resultados iniciales y los finales son prácticamente iguales. Esto ha podido deberse a la utilización de fichas para su evaluación; en las demás pruebas, al tratarse de evaluaciones en las que el alumnado utilizaba el material de Lego, las niñas/os se las tomaban como un juego y no como una tarea del aula. Así, se ha podido observar un mayor aprendizaje y diferencias significativas a la hora de comparar los resultados. Por último, debemos mencionar que durante las sesiones de intervención se pudo observar que el alumnado hizo uso de la comunicación a la hora de colocar los ladrillos durante la seriación (que se hizo en equipo). En casi todos los grupos siempre había uno o dos sujetos que coordinaban y verbalizaban por qué debían poner ese ladrillo en un lugar determinado. Los aspectos positivos que se pueden extraer de esta prueba son el trabajo en equipo y el desarrollo de la comunicación verbal frente a la toma de decisiones.

En la prueba 2, se han percibido mejoras en los resultados finales en comparación con los de diagnóstico. Esta prueba trabajaba diferentes conceptos que una parte de la muestra ya tenía adquiridos, pero el concepto que se quería trabajar con mayor interés era el de la cantidad. Para trabajar este concepto, es 
fundamental que la mayoría del alumnado tenga ya interiorizado la noción de número y que ya hayan trabajado la grafía de estos números, porque estos están directamente relacionados entre sí. Si no saben el número, no entenderán su grafía ni tampoco captarán la propia noción de grafía. Por todo ello, se han trabajado estos tres conceptos a la vez. Desde el principio de la prueba el alumnado ha mostrado interés hacía la realización de esta, pues los sujetos demandaban la utilización de los materiales de las sesiones de intervención para su uso a modo de juego y entretenimiento en otros momentos.

Para finalizar la valoración de las pruebas, cabe mencionar que la número 3 ha sido la que ha obtenido mejores resultados, así como la que más interés y motivación ha generado en el alumnado. Desde la prueba inicial de diagnóstico, el material ya despertó la curiosidad y el interés por la prueba en el alumnado. Al utilizar un material tan llamativo y diferente como es el set de bricks Lego Soft, los sujetos querían participar incluso en la preparación de las pruebas de evaluación y en la de las actividades de la sesiones de intervención. Esto puede confirmar la poca efectividad de la prueba 1 en comparación con esta, ya que el material de la primera prueba era muy básico y no tan llamativo. En cuento al motivo de que en cada sesión la construcción y la ubicación u orientación de la construcción fuera diferente, al cambiar de forma y orientación disminuía la probabilidad de que el alumnado memorizara las indicaciones relativas a las posiciones de la primera construcción. Por este motivo, al cambiar cada sesión, debían fijarse en la construcción y su posición con respecto a esta a fin de colocarse adecuadamente según la indicación que se les había dado.

\section{Bibliografía}

Carbonell, Jaume. 2006. La aventura de innovar: El cambio en la escuela. Madrid: Morata.

Garon, Denise, y Roland Filion. 1996. El sistema ESAR. Un método de análisis psicológico de los juguetes. Alicante: AIJU.

Lamoyi, Lourdes Beatriz. 2012. "La robótica Lego Mindstorms ${ }^{\circledR}$ : un recurso didáctico para fortalecer el pensamiento lógico matemático». Perspectivas Docentes 47: 12-17.

López, Irene. 2010. "El juego en la educación infantil y primaria». Autodidacta: Revista de la Educación en Extremadura 16: 19-37. 